\title{
Designing Interactive Systems for Balance Rehabilitation after Stroke
}

\author{
Héctor A. Caltenco \\ Anna Olsson \\ Afshin Aliyari \\ Charlotte Magnusson \\ Lund University \\ 22100 Lund, Sweden \\ hector@certec.lth.se \\ mte11aol@student.lu.se \\ afshin.aliyari@certec.Ith.se \\ charlotte@certec.Ith.se

\section{David McGookin} \\ Mikko Kytö \\ Aalto University \\ 02150 Espoo, Finland \\ david.mcgookin@aalto.fi \\ mikko.kyto@aalto.fi \\ Ingibjörg Hjaltadóttir \\ B. Hafsteinsdóttir \\ niversity of \\ Reykjavík, Iceland \\ t.hafsteinsdottir@umcutrecht.nl \\ helgaj@hi.is \\ ingibjar@landspitali.is
}

Permission to make digital or hard copies of part or all of this work for personal or classroom use is granted without fee provided that copies are not made or distributed for profit or commercial advantage and that

copies bear this notice and the full citation on the first page. Copyrights for third-party components of this work must be honored. For all other uses, contact the Owner/Author.

Copyright is held by the owner/author(s).

TEI '17, March 20-23, 2017, Yokohama, Japan

ACM 978-1-4503-4676-4/17/03.

ACM $978-1-4503-4676-4 / 17 / 03$.

\begin{abstract}
This paper presents four different tangible interactive prototypes designed to support the continuation of balance rehabilitation at home. The interactive prototypes are designed to provide a more enjoyable and experience when performing balance rehabilitation exercises. Early results from preliminary tests with stroke survivors and rehabilitation professionals are promising. The aim of the designs is to allow people at different levels of rehabilitation to engage into balance training and perform the exercises according to their current skills.
\end{abstract}

\section{Author Keywords}

User studies; stroke; activity; tangible interaction; codesign; participatory design.

\section{ACM Classification Keywords}

H.5.m. Information interfaces and presentation (e.g., $\mathrm{HCI}$ ): Miscellaneous

\section{Introduction}

Stroke is the leading cause of long-term disability in western countries [10] resulting in life-altering changes for both the stoke survivor and their closest family. Loss of balance is common among stroke-survivors. Difficulties with posture and balance make it difficult for persons to walk, and thus to leave the home to 
socialize or perform other outdoor tasks. After intensive rehabilitation in a dedicated rehabilitation setting, the majority of stroke survivors with residual impairments are discharged and then rely on informal caregivers (frequently close family members) [5].

Effective rehabilitation of stroke survivors is required to help them accomplish daily activities (cooking, washing, etc.) and lead an independent life. After discharge, much of this rehabilitation occurs at home, through performing different types of exercises and activities. Such as training in activities of daily living, strength training, cardio-vascular training, balance training, gait training and postural control [4]. However, training exercises can be difficult to integrate into the existing lives of stroke survivors. Exercises can be repetitive and difficult to perform correctly. Which leads to stroke survivors losing confidence and giving up on rehabilitation, losing the benefits it could provide. Thus, it is important to investigate how to better support the continuation of rehabilitation at home.

Donker et. al. [1] used interactive tiles for balance training during stroke rehabilitation sessions. They showed that providing real time visual feedback improved motivation and was helpful for balance rehablitation. Auditory feedback via sonification is also helpful for improving motoric skills [2]. Stienstra et. al. [8] studied how movement sonification improves proprioception during speedskating, if it is latency-free, and the richness of data details generated by the movement are preserved in the richness of sound parameters, such as dynamics, loudness, tempo, pitch and timbre. Other relevant work has proven the benefits of movement sonification on proprioception and awareness $[3,7]$.
Moreover, music and rhythm therapy are proven to help stroke survivors to increase the feeling of being connected to their own body. A study by Thornberg et. al. [9] shows that music and rhythm exercises were considered positively challenging for stroke survivors, while facilitating motor planning and coordination. Music is also a significant motivation factor while training which can give emotional pleasure [9]. Pleasant sounds tend to persuade the user into behavioural change [8].

The ACtivABLES research project is aimed at investigating the role interactive technologies can play for stroke survivors and support activities and training at home, in between formal therapy sessions or after discharge. Even though the ActivABLES project focuses on a range of different aspects of physical rehabilitation, the present paper presents different interactive prototypes designed to support the continuation of balance rehabilitation at home. The goal is to provide a more enjoyable and experience when performing balance rehabilitation exercises. We present early results from the initial testing of our prototypes.

\section{Interactive Tangible Prototypes}

Balance training and postural control can be performed in different ways. Active balance training happens when one must constantly move to maintain equilibrium.

Passive balance training is where one will stay in balance if one doesn't move. Balance can be performed in static surfaces or dynamic surfaces. Static surfaces are firm, uneven or compliant surfaces which do not move regardless of the location or amount of force applied to them. Dynamic surfaces will move or tilt if any external force is applied, disturbing its center of gravity. 


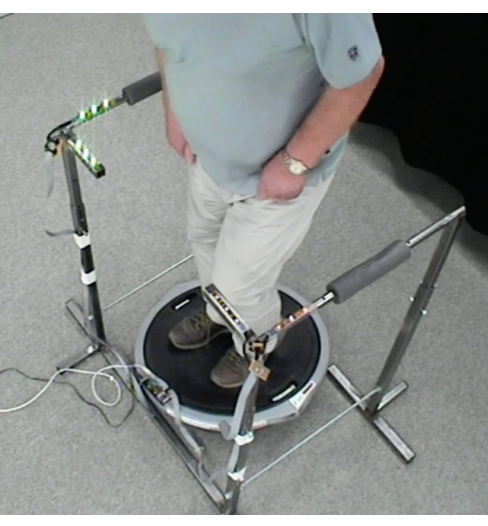

Figure 1. Stroke survivor using ActivBOSU, leaning slightly towards the right. Therefore the front-right and back-right indicators are lighted green.

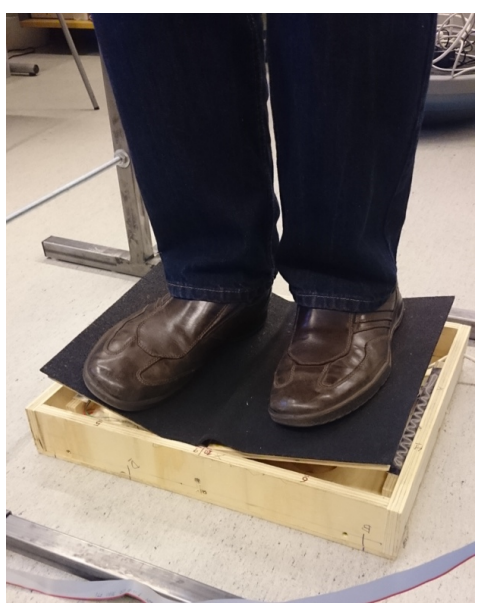

Figure 2. ActivPEDALS linked together by a rubber sheet. The same safety handle bars are used as in ActivBOSU.
In this section we present three different interactive prototypes designed to train passive and active balance while sitting or standing using static and dynamic surfaces. Performing exercises with these interactive objects could be done in different ways, depending on the preferences and motivation of the individuals. Due the benefits of music and rhythm therapy as well as the added motivation factor, the designed interactive balance-training devices are used to play/create music while performing rehabilitation exercises.

\section{ActivBOSU}

The ActivBOSU is a dynamic surface that can be used to train active or passive balance while standing. It consists of a BOSU balance board instrumented with an Arduino microcontroller, a 3-axis accelerometer which detects the tilt angle and direction. The safety support handle bars are equipped with RGB LED strips that provide visual feedback on the amount and direction of the tilt. All LED indicators are placed in the frontal half of the support handle bars so the user can see them without having to turn the head around (Figure 1). The Arduino UNO microcontroller sends the accelerometer signals to a computer running Ableton Live which produces different sound effects depending on the amount and direction of the tilt. Tilting the device affects both music and light simultaneously.

Two training programs were developed. In the active balance training program, if the user is standing still in a vertical position no light or sound are on. If the user is moving or standing still in tilted positions different sound landscapes and LEDs are activated, depending on the direction. The intensity of music and colour of light depends on how much the balance board is tilted. The light in the direction where the board is tilted is lighted up. In the "safe zone" when the balance board is just slightly tilted, the colour of the light is green, then yellow and when the light turns red the person shall be careful not to lose his/her balance.

In the passive balance training program, when the person is standing still and centred, soft classical music can be heard and no lights on. If the user tilts the platform, the music gradually gets distorted and the colour of the led-lights goes from green, to yellow, to red depending on how much the ActivBOSU tilts. The constructed prototype has only one degree of difficulty, but it is possible to inflate or deflate the BOSU ball to make it less or more stable, one can also change how sensitive the light/music feedback is with a new calibration.

During the development of the prototype the ergonomic aspects were considered. The plate where the feet are placed is suitable for all sizes and shapes of feet. The user is free to place his/her feet in an angle that feels comfortable and can easily change it without changing any settings. Stroke-survivors need to be able to hold on to the safety handles in order to minimize the risk of falling. The handles are designed so that they should be stable, comfortable to hold on to, fit into a normal home or local for rehabilitation, be movable and not leaving permanent marks in the floor. The handles are adjustable in height and distance from each other.

\section{ActivPEDALS}

ActivPEDALS is a variation of ActivBOSU. It is designed as two independent dynamic surfaces that can be used to train active or passive balance while standing, in addition of being able to train ankle flexion, extension, pronation and supination while standing or sitting. 

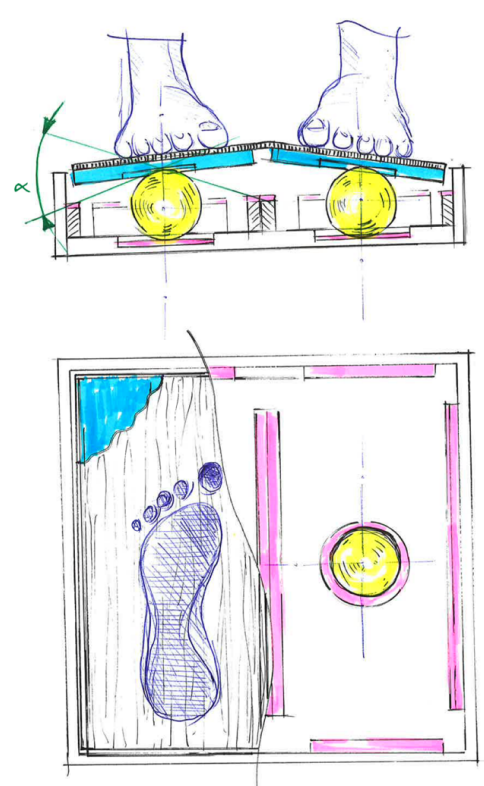

Figure 3. ActivPEDALS illustration. Two independent platforms (blue) standing over rubber balls (yellow) and eight pressure sensors (pink) that detect contact between platforms and the box. Two pressure sensors under the rubber balls detect the weight distribution of the person standing on the prototype.
ActivPEDALS consists of a wooden box with two independent platforms standing over rubber balls fixed at the bottom of the box, which allow the platforms to tilt towards the edges of the box. The platforms can be link-together to act as a single large platform instead (Figure 2). Each platform is instrumented with an accelerometer that detects the amount and direction of tilt. Eight Pressure sensors detect if there is contact between the platform edges and the box (Figure 3 ).

An Arduino UNO microcontroller sends the signals from both accelerometers and the eight multiplexed pressure sensors to a computer running Ableton Live which produces different sound effects depending on the amount and direction of the tilt of each platform and the amount of pressure on the sensors. The tilt and contact signals affects different sound parameters and lights at the same time. One can increase or decrease the stability of the platforms by easily exchanging the balls inside the box for ones with larger or smaller diameter. One can also change how sensitive the light/music feedback is with a new calibration.

ActivPEDALS can be used to train balance standing with one foot over each platform, in which case it has the same training programs for training while aiming to stand still and aiming to keep balance while actively leaning towards different sides. It provides extra sound layers and effects linked to the accelerometer signal of the second platform and different drum sounds for each of the pressure sensors. Lights are off if the platforms are centred and balanced. If a platform is slightly tilted the corresponding LEDs will light up green, or red if a platform activates a pressure sensor. The intensity of the light is mapped to the amount of tilt or pressure.
ActivCUSHION and ActivFOAM

ActivCUSHION and ActivFOAM are a static compliantsurfaces that can be used to train active balance while sitting or standing. These kinds of surface and training are used at the beginning of rehabilitation, when is difficult to keep balance when training in dynamic surfaces. They are designed to challenge balance and provide postural training.

ActivFOAM consists of a soft-compliant foam board for standing on, while ActivCUSHION consists of an inflatable cushion for seating on. Both of them are covered with a sandwich design of pressure-sensing fabric material (FlexTiles [6]). Which consists of two electrode layers of zebra fabric and a force sensitive layer of piezo resistive fabric in between (Figure 4).

The two layers of fabric are oriented orthogonal to each-other to form a matrix of pressure sensors with a resolution of $16 \times 16$ and $16 \times 24$ sensors for ActivCUSHION and ActivFOAM respectively. All rows and columns in the sensor matrix are connected to two 8-channel analog multiplexers (74HC4051) with pulldown $20 \mathrm{Kohm}$ resistors. The multiplexed rows go to the analog inputs of an Arduino microcontroller. The multiplexed columns are connected to the digital output pins, which sends $5 \mathrm{~V}$ signals to one column at a time.

The working principle of ActivFOAM and ActivCUSHION is different from that one of ActivBOSU and

ActivPEDALS, but the applications are similar. Instead of visual feedback using LED strips, the visual feedback is given to the user via a computer display. The corresponding pressure matrix is displayed on the screen as an array of black squares that change gradually towards white depending on how much 


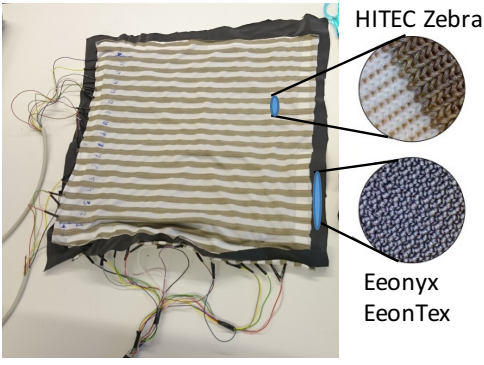

Figure 4. Pressure sensor matrix cover for ActivFOAM and

ActivCUSHION. Sandwich design of two electrode layers of zebra fabric and a force sensitive layer of EeonTex piezo resistive fabric in between.

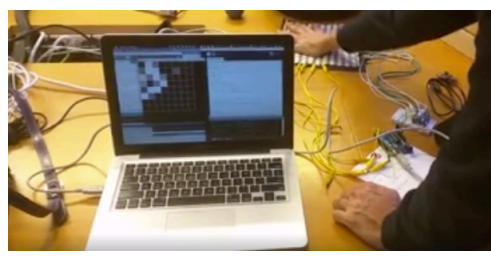

Figure 5. Testing visual feedback of the pressure sensor matrix cover for ActivFOAM and ActivCUSHION. pressure is registered in each sensor (Figure 5). The sound feedback is the same as in the other prototypes, but instead of using the tilting of the platforms, the center of gravity of the shape(s) registered by the pressure sensor matrix is used. If more pressure is registered in e.g., the left foot, then the sound effect will play as if the user was tilting towards the left. ActivCUSHION follows the same principle, but for practicing balance while sitting.

\section{Preliminary Evaluations}

A preliminary evaluation of the prototypes was performed by three stroke survivors and by a group of rehabilitators and physical therapists. The preliminary tests started with a short interview to gather background information about their lives before and after the stroke, their rehabilitation, what has worked well and what can be improved, goals, interests, and motivation to perform exercises. After the interviews, participants tested the interactive tangibles using the passive balance and active balance training programs. Afterwards, a short discussion about the test was done.

\section{ActivBOSU}

The results from the preliminary tests of ActivBOSU with stroke-survivors were promising. The prototype was described as uncomplicated, flexible, motivating and stimulating by one stroke survivor. This person said it was more fun to train the balance when music was included, which lead to higher motivation to train. It was a general agreement by stroke survivors and rehabilitators that ActivBOSU needs to have more music or sound options and different training-programs to make the training fun and motivating over time. Ledlights was included in the support handle bars to give feedback on how the user tilted the balance-board and it helped the user to find a straighter body-position. However, it was hard for one of the stroke survivors to look down to see the lights. Whenever he tried to look at them, he felt he lost his balance. Visual feedback should be provided at eye-level to avoid this problem. The third stroke survivor felt the feedback was overwhelming, and would have preferred to be able to use it without any additional feedback - at least initially. This reflects the well-known fact that stroke survivors are a very diverse population.

\section{ACtivPEDALS}

One of the design ideas of ActivePEDALS was to make the active balance training safer and easier, since the range of motion is lower than the one of ActivBOSU. But in fact, both stroke survivors and rehabilitators found it harder to keep balance with ActivePEDALS. We believe, this is mostly due to the independent plates. However, the rehabilitators found it potentially very useful to rehabilitate ankle flexion/extension and pronation/supination while sitting. Moreover, it has good potential for more complex musicalization, due to the independent plates and the added pressure sensors. And it could be a more challenging option for rehabilitees with better balance.

\section{ActivFOAM and ActivCUSHION}

During these tests, auditive feedback was not available and only visual feedback was demonstrated. ActivFOAM was seen by the rehabilitators as promising, since balance rehabilitation of all stroke survivors starts with similar foam-mats and having one that could provide visual and musical feedback for balance and posture while standing and sitting was something they looked forward to. 


\section{Conclussions}

In general, continuing to train balance at home, after discharge, is important for stroke survivors to keep their functional level. Incorporating music into interactive tangibles for balance rehabilitation can potentially help the motivation and make the training more enjoyable and fun. Moreover, having a set of different interactive tangibles to train passive and active balance, and having static and dynamic surfaces will allow people at different levels of rehabilitation to engage into balance training and perform the exercises.

\section{Acknowledgements}

We would like to extend our gratitude to all the stroke survivors in Sweden and health care professionals in Finland taking part in the research. The authors also gratefully acknowledge NordForsk for the support given to the project ActivABLES, as well as the EU for the support of the related project 689947 STARR.

\section{References}

1. V Donker, P Markopoulos, and B Bongers. 2015. REHAP Balance Tiles: A modular system supporting balance rehabilitation. Pervasive Computing Technologies for Healthcare (PervasiveHealth), 2015 9th International Conference on, 201-208. http://doi.org/10.4108/icst.pervasivehealth.2015.2 59278

2. Alfred O Effenberg. 2007. Movement Sonification: Motion perception, behavioral effects and functional data. Proceedings of the 2 nd International Workshop on Interactive Sonification (ISon 2007), 1-4.

3. Frank Feltham and Lian Loke. 2014. The slow floor: increasing creative agency while walking on an interactive surface. Proceedings of the 8 th International Conference on Tangible, Embedded and Embodied Interaction: 105-112.

4. Hanne Kaae Kristensen, Charlotte Ytterberg, Dorrie
Lee Jones, and Hans Lund. 2016. Research-based evidence in stroke rehabilitation: an investigation of its implementation by physiotherapists and occupational therapists. Disability and rehabilitation: $1-11$.

http://doi.org/10.3109/09638288.2016.1138550 B J Lutz, M E Young, K J Cox, C Martz, and K R

Creasy. 2011. The crisis of stroke: experiences of patients and their family caregivers. Topics in stroke rehabilitation 18, 6: 786-797. http://doi.org/10.1310/tsr1806-786 ;

6. Patrick Parzer, Kathrin Probst, Teo Babic, et al. 2016. FlexTiles: A Flexible, Stretchable, Formable, Pressure-Sensitive, Tactile Input Sensor. Proceedings of the 2016 CHI Conference Extended Abstracts on Human Factors in Computing Systems, ACM, 3754-3757.

7. Prashant Srinivasan, David Birchfield, Gang Qian, and Assegid Kidané. 2005. A pressure sensing floo for interactive media applications. ACE '05:

Proceedings of the 2005 ACM SIGCHI International Conference on Advances in computer entertainment technology, 278-281. http://doi.org/http://doi.acm.org/10.1145/117847 7.1178526

8. Jelle Stienstra, C.J. Overbeeke, and Stephan Wensveen. 2011. Embodying Complexity Through Movement Sonification : Case Study on

Empowering the Speed-skater. Proceedings of the 9th ACM SIGCHI Italian Chapter International Conference on Computer-Human Interaction: Facing Complexity: 39-44.

http://doi.org/10.1145/2037296.2037310

9. Kerstin Thornberg, Staffan Josephsson, and Ingrid Lindquist. 2014. Experiences of participation in rhythm and movement therapy after stroke. Disability and rehabilitation 36, 22: 1869-74.
http://doi.org/10.3109/09638288.2013.876107

10. C D Wolfe. 2000. The impact of stroke. British Medical Bulletin 56, 2: 275-286.

http://doi.org/10.1258/0007142001903120 